\title{
Biologia e tabela de vida de fertilidade do pulgão-preto em cultivares de videira
}

\author{
Cléber Antonio Baronio(1), Simone Andzeiewski(2), Uemerson Silva da Cunha( ${ }^{(1)}$ e Marcos Botton ${ }^{(2)}$
}

\begin{abstract}
(1)Universidade Federal de Pelotas, Departamento de Fitossanidade, Caixa Postal 354, CEP 96010-900 Pelotas, RS, Brasil. E-mail: cleber.baronio@hotmail.com, uscunha@yahoo.com.br ${ }^{(2)}$ Embrapa Uva e Vinho, Laboratório de Entomologia, Rua Livramento, n 515, Caixa Postal 130, CEP 95700-000 Bento Gonçalves, RS, Brasil. E-mail: simoneandzeiewski@yahoo.com.br, marcos.botton@embrapa.br
\end{abstract}

Resumo - O objetivo deste trabalho foi avaliar a biologia e a tabela de vida de fertilidade do pulgão-preto da videira [Aphis illinoisensis (Hemiptera: Aphididae)], em mudas das cultivares de Vitis labrusca Bordô, e de $V$. vinifera Cabernet Franc, Itália e Moscato Bianco. O experimento foi conduzido em 50 minigaiolas de confinamento, com um inseto em cada gaiola por tratamento, fixadas em mudas de videira mantidas em câmara de crescimento do tipo fitotron (a $25 \pm 1{ }^{\circ} \mathrm{C}$, umidade relativa de $75 \pm 10 \%$ e fotófase de 14 horas). Avaliaram-se diariamente a duração e a viabilidade ninfal, a fecundidade e a longevidade do período reprodutivo da espécie. O pulgão-preto da videira completou o ciclo biológico nas mudas das mencionadas cultivares, com duração

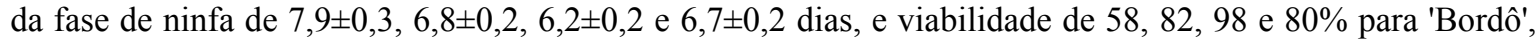
'Cabernet Franc', 'Itália' e 'Moscato Bianco', respectivamente. 'Cabernet Franc' e 'Moscato Bianco' foram mais favoráveis ao desenvolvimento do pulgão-preto, com base na tabela de vida de fertilidade, com 51,3 e 55,6 descendentes por fêmea, por geração, respectivamente. 'Bordô' foi a menos adequada ao desenvolvimento do afídeo, com 12,55 descendentes por fêmea, por geração, o que indica resistência do tipo antibiose ou não preferência do inseto pela cultivar.

Termos para indexação: Aphis illinoisensis, Vitis labrusca, Vitis vinifera, antibiose, fecundidade, manejo integrado de pragas.

\section{Biology and fertility life table of grapevine aphid on grape cultivars}

\begin{abstract}
The objective of this work was to evaluate the biology and fertility life table of the grapevine aphid [Aphis illinoisensis (Hemiptera: Aphididae)] on seedlings of the grape cultivars of Vitis labrusca, Bordô, and of $V$. vinifera, Cabernet Franc, Italy, and Moscato Bianco. The experiment was carried out in 50 small-cage confinement, with one insect in each small-cage per treatment, fixed in grapevine seedlings kept in a phytotrontype growth chamber (at $25 \pm 1{ }^{\circ} \mathrm{C}, 75 \pm 10 \%$ relative humidity, and 14-hour photophase). Duration and viability of nymphal stages, fecundity, and longevity of the species reproductive period were daily evaluated. Grapevine aphid completed its life cycle in grape seedlings of the mentioned cultivars, with nymphal stage duration of $7.9 \pm 0.3,6.8 \pm 0.2,6.2 \pm 0.2$ and $6.7 \pm 0.2$ days, and viability of 58, 82, 98, and 80\%, for 'Bordô', 'Cabernet Franc', 'Italia', and 'Moscato Bianco', respectively. 'Cabernet Franc' and 'Moscato Bianco' were more favorable to the grapevine aphid development, based on the fertility life table, with 51.3 and 55.6 offspring per female, per generation, respectively. 'Bordô' was the less suitable for this aphid development, with 12.55 offspring per female, per generation, which indicates a antibiosis-type resistance or insect nonpreference for this cultivar.
\end{abstract}

Index terms: Aphis illinoisensis, Vitis labrusca, Vitis vinifera, antibiosis, fecundity, integrated pest management.

\section{Introdução}

A viticultura é uma importante atividade econômica e social para o Brasil, seja pelo número de empregos originados diretamente do cultivo ou indiretamente das atividades industriais, de processamento e de turismo associadas à cultura (Mello, 2013). Nas áreas vitivinícolas, as pragas e doenças constituem alguns dos maiores obstáculos à expansão do cultivo da videira, o que afeta tanto a quantidade como a qualidade do produto final (Formolo et al., 2011). Nos últimos anos, tem ocorrido um aumento significativo da incidência do pulgão-preto da videira [Aphis illinoisensis Shimer, 1866 (Hemiptera: Aphididae)] em vinhedos da região Sul do Brasil. A alimentação do afídeo provoca redução do desenvolvimento vegetativo, formação de bifurcações e encarquilhamento de folhas novas e brotos e, em situações de elevada infestação, levam à 
queda de bagas no período entre a floração e a colheita (Blackman \& Eastop, 2006). A espécie é originária da América do Norte, porém, tem-se expandido mundialmente (Havelka et al., 2011; El-Gantiry et al., 2012).

No Brasil, o primeiro relato sobre $A$. illinoisensis em videira foi registrado em São Paulo, em uvas finas de mesa Vitis vinifera 'Itália' (Kuniyuki et al., 1995). Mesmo com a recente expansão da praga e os danos causados, poucas informações sobre a biologia de $A$. illinoisensis na cultura estão disponíveis (Moraiti et al., 2012), principalmente quanto ao seu comportamento em cultivares de $V$. labrusca, predominantes nos vinhedos brasileiros.

O controle da espécie tem sido necessário, principalmente quando o ataque ocorre nos ponteiros de plantas em fase de formação e logo após a floração, quando os pulgões migram também para os cachos (Zaaqiq, 2007). Informações sobre o comportamento biológico da espécie, em diferentes cultivares de videira, são escassas, embora sejam imprescindíveis para a implementação de um programa de manejo integrado.

O objetivo deste trabalho foi avaliar a biologia e tabela de vida de fertilidade do pulgão-preto da videira [Aphis illinoisensis (Hemiptera: Aphididae)], em mudas das cultivares de Vitis labrusca Bordô, e de Vitis vinifera Cabernet Franc, Itália e Moscato Bianco.

\section{Material e Métodos}

O estudo da biologia de $A$. illinoisensis foi conduzido na Embrapa Uva e Vinho, em Bento Gonçalves, RS, com uso de uma câmara de crescimento do tipo fitotron Percival Boone, modelo 50036 (4 m de comprimento x $3 \mathrm{~m}$ de largura $\mathrm{x} 3,5 \mathrm{~m}$ de altura), à temperatura de $25 \pm 1{ }^{\circ} \mathrm{C}$, umidade relativa de $75 \pm 10 \%$ e fotófase de 14 horas.

Os parâmetros biológicos do pulgão-preto da videira foram determinados em mudas de V. labrusca 'Bordô' (utilizada para a produção de sucos concentrados e vinhos tintos comuns) e de $V$. vinifera 'Cabernet Franc' (destinada à elaboração de vinho tinto seco fino), 'Itália' (uva fina de mesa) e 'Moscato Bianco' (usado para a elaboração de vinho branco fino). Estas cultivares foram escolhidas por representar os principais grupos de videiras cultivados na região, além da disponibilidade de material vegetativo para a condução do trabalho.
As mudas foram produzidas com uso de estacas coletadas em matrizeiro da Embrapa Uva e Vinho, enraizadas em baldes com solução de ácido indolbutírico (AIB) à concentração de $2 \mathrm{mg} \mathrm{L}^{-1}$ por 24 horas. Após este período, as estacas foram mantidas em câmara refrigerada por duas semanas, para homogeneizar a brotação até serem transplantadas para os vasos. 'Cabernet Franc' e 'Itália' foram adquiridas de viveiristas, enxertadas sobre porta-enxerto 'Paulsen 1103', enquanto as mudas de 'Bordô' e 'Moscato Bianco' foram produzidas em pé-franco.

O trabalho foi conduzido com adaptação do método utilizado por Moraiti et al. (2012). Para cada cultivar de videira, foram utilizadas 20 mudas com um ano de idade, transplantadas em vasos com capacidade volumétrica de $2 \mathrm{~L}$, tendo-se utilizado terra vermelha como substrato, composto Mecplant e vermiculita à proporção de $3: 2: 1$, autoclavados a $140^{\circ} \mathrm{C}$ por $50 \mathrm{~min}$, para prevenir a contaminação por fungos de solo.

Adultos de pulgão-preto provenientes da criação de manutenção, mantidos em mudas de 'Cabernet Sauvignon' por oito meses, foram transferidos para folhas das cultivares a serem avaliadas. Em cada folha, foram colocadas três fêmeas adultas, com idade entre 8 e 13 dias, mantidas no interior de uma minigaiola confeccionada com recipiente de plástico de $2,5 \mathrm{~cm}$ de diâmetro x $1 \mathrm{~cm}$ de altura, com dois orifícios circulares de $0,5 \mathrm{~cm}$ de diâmetro vedados com tecido do tipo voile no fundo do recipiente. Para evitar a fuga dos insetos, uma espuma de $0,5 \mathrm{~cm}$ de espessura por $4 \mathrm{~cm}$ de diâmetro, com um orifício circular do mesmo diâmetro da minigaiola, foi fixada à base do recipiente e ajustada às folhas, sem danificá-las. Para fixar as minigaiolas rentes às folhas, utilizou-se uma presilha, que continha na outra extremidade outra circunferência de espuma de $4 \mathrm{~cm}$ de diâmetro, com a finalidade de evitar o dobramento da folha a ser colocada entre a minigaiola e a outra extremidade da presilha. As minigaiolas permaneceram suspensas com o auxílio de um fio de náilon de $15 \mathrm{~cm}$ de comprimento, preso a uma haste de ferro vertical, para não danificar o pecíolo da folha e para evitar sua quebra com o peso. A cada planta, foram fixadas duas ou três minigaiolas.

Decorridas 24 horas após a infestação, as fêmeas e as ninfas excedentes foram retiradas, tendo-se mantido uma ninfa por minigaiola. As ninfas foram avaliadas diariamente, com auxílio de uma lupa manual (10X) até a passagem para a fase adulta. Para cada cultivar, 
foram estabelecidas 50 repetições (minigaiolas), tendo-se adaptado o método proposto por Moraiti et al. (2012), que utilizaram 30 plantas, com um inseto por tratamento, e acompanharam os indivíduos até a fase adulta. A determinação dos instares ninfais foi realizada com base na presença das exúvias liberadas pelas ninfas.

Para avaliar a fecundidade e longevidade dos adultos, as ninfas que se desenvolveram agrupadas nas plantas de cada cultivar avaliada, ao atingir o quarto instar, foram colocadas individualmente nos ponteiros de mudas da mesma cultivar para serem avaliadas até a fase adulta, tendo-se registrado diariamente os parâmetros biológicos dos adultos (período pré-reprodutivo, reprodutivo, pós-reprodutivo e fecundidade), com 20 repetições por cultivar.

Os dados de biologia do pulgão-preto da videira foram analisados, com a elaboração da tabela de vida de fertilidade sobre as diferentes cultivares. Estimaram-se: o intervalo médio entre gerações $(\mathrm{T})$; a taxa líquida de reprodução $\left(\mathrm{R}_{\mathrm{o}}\right)$; a taxa intrínseca de crescimento $\left(\mathrm{r}_{\mathrm{m}}\right)$; a razão finita de aumento $(\lambda)$; e o tempo necessário para a população duplicar em número de indivíduos (TD), com o método "jackknife" (Maia \& Luiz, 2006).

Para avaliar a influência das cultivares sobre o desenvolvimento biológico e o aumento da população de pulgão-preto, realizou-se o cálculo do índice de potencial reprodutivo corrigido $\mathrm{PRC}=\left(\begin{array}{rrr}r s & \mathrm{x}\end{array}\right)^{\mathrm{n}}$ (Vendramin \& Fancelli, 1988), em que: rs (razão sexual) $=\mathrm{n}^{\mathrm{o}}$ de fêmeas $/ \mathrm{n}^{\mathrm{o}}$ de adultos; A (número de adultos aptos à reprodução) $=$ número de ninfas/fêmea $\times$ viabilidade da fase ninfal; $n$ é o número de gerações do inseto em 30 dias.

Os dados foram analisados quanto à normalidade, com o teste de Shapiro-Wilk (1965), e de homogeneidade da variância dos erros (Bartlett, 1937; Hartley, 1950). Como instrumento de discriminação dos tratamentos, as médias de 'Bordô', 'Cabernet Franc', 'Itália' e 'Moscato Bianco' foram comparadas pelo teste de Tukey, a $5 \%$ de probabilidade, com o programa Statistica 7.0 (Statsoft, Tulsa, EUA). A longevidade dos insetos foi calculada com o uso do modelo de distribuição de Weibull (Sgrillo, 1982).

\section{Resultados e Discussão}

O pulgão-preto da videira apresentou quatro instares, em todas as cultivares de videira avaliadas, e diferenças significativas em seus parâmetros biológicos, conforme a cultivar sobre a qual o inseto foi criado (Tabela 1). Para o primeiro instar, a menor duração do período foi observada na 'Cabernet Franc', 'Moscato Bianco' e 'Itália' que diferiram da 'Bordô' (2,17 dias). A viabilidade do primeiro instar foi de $100 \%$ na 'Cabernet Franc', 'Itália' e 'Moscato Bianco', que diferiram dos $84 \%$ registrados na 'Bordô'.

No segundo instar, não houve diferença significativa entre as cultivares quanto à duração do período, que variou de 1,41 ('Itália') a 1,71 dias ('Moscato Bianco'). No terceiro instar, não houve diferença significativa da duração do período entre as cultivares, em que a 'Itália' apresentou maior viabilidade, juntamente com a 'Cabernet Franc' e 'Moscato Bianco', que diferiram da 'Bordô'.

A maior duração do quarto instar foi observada na 'Bordô' e 'Cabernet Franc' que não diferiram da 'Itália' e 'Moscato Bianco' (Tabela 1). Neste instar, a viabilidade ninfal sobre a 'Bordô' foi menor e diferiu da 'Cabernet Franc', 'Itália' e 'Moscato Bianco'.

A duração média da fase ninfal apresentou diferença significativa entre as cultivares, que variou 6,20 a 7,90 dias na 'Itália' e 'Bordô', respectivamente (Tabela 2). Moraiti et al. (2012) não observaram diferenças significativas na fase ninfal, nas cultivares de $V$. vinifera Angiorgitiko e Soultanina, com durações de 5,57 e 5,87 dias, respectivamente, à temperatura de $23^{\circ} \mathrm{C}$. No presente trabalho, a maior duração da fase ninfal foi observada em $V$. labrusca, enquanto as

Tabela 1. Duração e viabilidade da fase de ninfa do pulgão-preto da videira (Aphis illinoisensis), em cultivares de videira, mantidas em câmara climatizada (a $25 \pm 1^{\circ} \mathrm{C}$, $75 \pm 10 \%$ de umidade relativa e fotófase de 14 horas) do tipo fitotron $^{(1)}$.

\begin{tabular}{lcccc}
\hline Instares & \multicolumn{4}{c}{ Cultivar } \\
\cline { 2 - 5 } & 'Bordô' & 'Cabernet Franc' & 'Itália' & 'Moscato Bianco' \\
\hline \multicolumn{5}{c}{ Duração (Dias) } \\
I & $2,17 \pm 0,20 \mathrm{~b}$ & $1,42 \pm 0,09 \mathrm{a}$ & $1,62 \pm 0,09 \mathrm{a}$ & $1,56 \pm 0,13 \mathrm{a}$ \\
II & $1,58 \pm 0,12 \mathrm{a}$ & $1,64 \pm 0,11 \mathrm{a}$ & $1,41 \pm 0,10 \mathrm{a}$ & $1,71 \pm 0,14 \mathrm{a}$ \\
III & $1,90 \pm 0,17 \mathrm{a}$ & $1,84 \pm 0,12 \mathrm{a}$ & $1,47 \pm 0,10 \mathrm{a}$ & $1,76 \pm 0,11 \mathrm{a}$ \\
IV & $2,00 \pm 0,15 \mathrm{a}$ & $1,95 \pm 0,09 \mathrm{a}$ & $1,71 \pm 0,11 \mathrm{a}$ & $1,90 \pm 0,10 \mathrm{a}$ \\
Total & $7,90 \pm 0,26 \mathrm{~b}$ & $6,83 \pm 0,19 \mathrm{a}$ & $6,20 \pm 0,15 \mathrm{a}$ & $6,68 \pm 0,24 \mathrm{a}$ \\
\hline \multicolumn{5}{c}{ Viabilidade $(\%)$} \\
I & $84,00 \pm 5,24 \mathrm{~B}$ & $100,00 \pm 0,00 \mathrm{~A}$ & $100,00 \pm 0,00 \mathrm{~A}$ & $100,00 \pm 0,00 \mathrm{~A}$ \\
II & $76,00 \pm 6,10 \mathrm{~B}$ & $94,00 \pm 3,39 \mathrm{~A}$ & $98,00 \pm 2,00 \mathrm{~A}$ & $96,00 \pm 2,80 \mathrm{~A}$ \\
III & $60,00 \pm 7,00 \mathrm{~B}$ & $86,00 \pm 4,96 \mathrm{~A}$ & $98,00 \pm 2,00 \mathrm{~A}$ & $92,00 \pm 3,88 \mathrm{~A}$ \\
IV & $58,00 \pm 7,05 \mathrm{~B}$ & $82,00 \pm 5,49 \mathrm{~A}$ & $98,00 \pm 2,00 \mathrm{~A}$ & $80,00 \pm 5,71 \mathrm{~A}$ \\
\hline (1)Média \pm EPM seguida por letras iguais, nas linhas, não diferem pelo teste
\end{tabular}
de Tukey, a 5\% de probabilidade. 
menores durações foram registradas em $V$. vinifera. As cultivares Cabernet Franc, Itália e Moscato Bianco apresentaram as maiores viabilidades e diferiram da V. labrusca 'Bordô'. Os dados observados em $V$. vinifera corroboram os encontrados por Moraiti et al. (2012), que obtiveram viabilidade do inseto entre 85 e $97 \%$ nas cultivares de $V$. vinifera estudadas. No entanto, Bertin et al. (2013) observaram maior período ninfal de Dismicoccus brevipes na 'Niágara Rosada' ( $V$. labrusca) do que na 'Itália' ( $V$. vinifera).

A maior duração da fase ninfal observada na 'Bordô', aliada à alta taxa de mortalidade e menor fecundidade, pode estar relacionada à ocorrência de resistência por antibiose ou por antixenose (não preferência) desta cultivar e é característica de V. labrusca (Valadão et al., 2012). Outra possível explicação pode ser a menor qualidade nutricional (Bertin et al., 2013).

Em relação à fase adulta do pulgão-preto, fêmeas alimentadas em mudas de 'Bordô' apresentaram maior período pré-reprodutivo, superiores aos observados na 'Cabernet Franc' e 'Moscato Bianco', em que a duração do período pré-reprodutivo não diferiu da 'Itália'.

O período reprodutivo do pulgão-preto foi maior na 'Moscato Bianco', que diferiu significativamente da 'Bordô', da 'Cabernet Franc' e da 'Itália' . Estes resultados diferiram dos obtidos por Moraiti et al. (2012), que observaram período reprodutivo médio de 11,40 a 13,60 dias nas principais cultivares de $V$. vinifera produzidas na Grécia.

O período pós-reprodutivo do inseto não diferiu entre as cultivares de videira avaliadas, tendo variado de 1,25 a 2,05 dias (Tabela 2). Este período foi inferior ao encontrado por Moraiti et al. (2012), que obtiveram maior período de sobrevivência dos adultos após encerrarem a reprodução, com variação média de 6,00 e 9,70 dias em cultivares de $V$. vinifera.

Quanto à duração do período adulto do pulgão-preto da videira, as fêmeas em ciclo de desenvolvimento que se alimentaram da 'Moscato Bianco' foram as mais longevas e diferiram das que se alimentaram da 'Bordô', da 'Cabernet Franc' e da 'Itália' (Figura 1 e Tabela 2).

A longevidade observada na 'Moscato Bianco' foi superior ao encontrado por Moraiti et al. (2012), que registraram longevidade de 19,70 dias nesta cultivar. Nas demais cultivares de $V$. vinifera avaliadas, os autores observaram, em média, longevidade maior que a da 'Moscato Bianco', de 19,80 a 23,30 dias, à temperatura de $23^{\circ} \mathrm{C}$.

Com a análise da curva de sobrevivência dos adultos de pulgão-preto da videira, observa-se que aos 25 dias de idade, a sobrevivência dos indivíduos alimentados com a 'Moscato Bianco' foi superior à observada na 'Bordô', na 'Cabernet Franc' e na 'Itália' (Figura 1). Moraiti et al. (2012) registraram elevada sobrevivência dos insetos até o vigésimo dia de vida e, apenas na cultivar Savvatiano, foram registrados adultos até os 24 dias de idade, com elevada mortalidade nos dias subsequentes. A fecundidade variou conforme a cultivar de videira em que os pulgões se alimentaram. A fecundidade diária dos insetos diferiu entre as cultivares e foi menor na 'Bordô', intermediária na 'Itália' e na 'Moscato Bianco' e maior na 'Cabernet Franc' (Tabela 2). As menores fecundidades totais foram observadas na 'Bordô' e na 'Itália' que diferiram da 'Cabernet Franc' e da 'Moscato Bianco'. Os resultados observados para 'Cabernet Franc' e 'Moscato Bianco' foram semelhantes aos encontrados por Moraiti et al. (2012), que obtiveram fecundidade média de 58,30 a 64,30 ninfas por fêmea, para as cultivares de $V$. vinifera estudadas. De acordo

Tabela 2. Parâmetros biológicos do pulgão-preto da videira (Aphis illinoisensis), em cultivares de videira, em câmara climatizada (a $25 \pm 1^{\circ} \mathrm{C}, 75 \pm 10 \%$ de umidade relativa e fotófase de 14 horas) do tipo fitotron ${ }^{(1)}$.

\begin{tabular}{|c|c|c|c|c|c|}
\hline \multirow[t]{2}{*}{ Parâmetro } & \multicolumn{4}{|c|}{ Cultivar } & \multirow{2}{*}{$\begin{array}{l}\text { CV } \\
(\%) \\
\end{array}$} \\
\hline & 'Bordô' & 'Cabernet Franc' & 'Itália' & 'Moscato Bianco' & \\
\hline Duração da fase ninfal (dias) & $7,90 \pm 0,26 b$ & $6,83 \pm 0,19 \mathrm{a}$ & $6,20 \pm 0,15 a$ & $6,68 \pm 0,24 a$ & 21,65 \\
\hline Viabilidade da fase ninfal (\%) & $58,00 \pm 7,05 b$ & $82,00 \pm 5,49 a$ & $98,00 \pm 2,00 \mathrm{a}$ & $80,00 \pm 5,71 \mathrm{a}$ & 48,00 \\
\hline Período pré-reprodutivo (dias) & $2,60 \pm 0,32 b$ & $1,40 \pm 0,15 \mathrm{a}$ & $1,90 \pm 0,23 \mathrm{ab}$ & $1,60 \pm 0,15 \mathrm{a}$ & 53,40 \\
\hline Período reprodutivo (dias) & $8,25 \pm 1,21 \mathrm{a}$ & $10,75 \pm 1,38 \mathrm{a}$ & $8,5 \pm 1,64 a$ & $17,45 \pm 2,15 b$ & 65,07 \\
\hline Período pós-reprodutivo (dias) & $1,40 \pm 0,37 \mathrm{a}$ & $1,70 \pm 0,24 \mathrm{a}$ & $1,25 \pm 0,16 \mathrm{a}$ & $2,05 \pm 0,44 \mathrm{a}$ & 90,03 \\
\hline Longevidade (dias) & $20,25 \pm 1,29 a$ & $20,70 \pm 1,53 \mathrm{a}$ & $17,90 \pm 1,64 \mathrm{a}$ & $27,85 \pm 2,29 b$ & 36,52 \\
\hline Fecundidade diária (ninfas por dia) & $1,58 \pm 0,15 \mathrm{a}$ & $5,22 \pm 0,36 \mathrm{c}$ & $2,62 \pm 0,15 b$ & $3,34 \pm 0,23 b$ & 33,69 \\
\hline Fecundidade total (ninfas por fêmea) & $12,55 \pm 2,06 \mathrm{~b}$ & $51,30 \pm 5,86 \mathrm{a}$ & $22,75 \pm 4,53 b$ & $55,60 \pm 7,26 \mathrm{a}$ & 66,50 \\
\hline
\end{tabular}

${ }^{(1)}$ Média \pm EPM seguida por letras iguais, nas linhas, não diferem pelo teste de Tukey, a 5\% de probabilidade. 
com Awmack \& Leather (2002), a fecundidade é o melhor parâmetro biológico para indicar a qualidade de um determinado hospedeiro sobre a reprodução de insetos sugadores; assim, 'Cabernet Franc' e 'Moscato Bianco' podem ser consideradas as mais favoráveis cultivares ao desenvolvimento do pulgão-preto da videira.

$\mathrm{O}$ índice potencial reprodutivo corrigido (PRC) do pulgão-preto da videira foi superior na 'Moscato Bianco' que, após quatro gerações, foi de $1.323,20 \times 10^{5}$, seguida da 'Itália' $\left(742,70 \times 10^{5}\right)$ e da 'Cabernet Franc' $\left(402,23 \times 10^{5}\right)$, ao longo do período de 30 dias. Quanto à V. labrusca 'Bordô', o PRC foi inferior ao das cultivares de $V$. vinifera avaliadas, com $0,10 \times 10^{5}$.
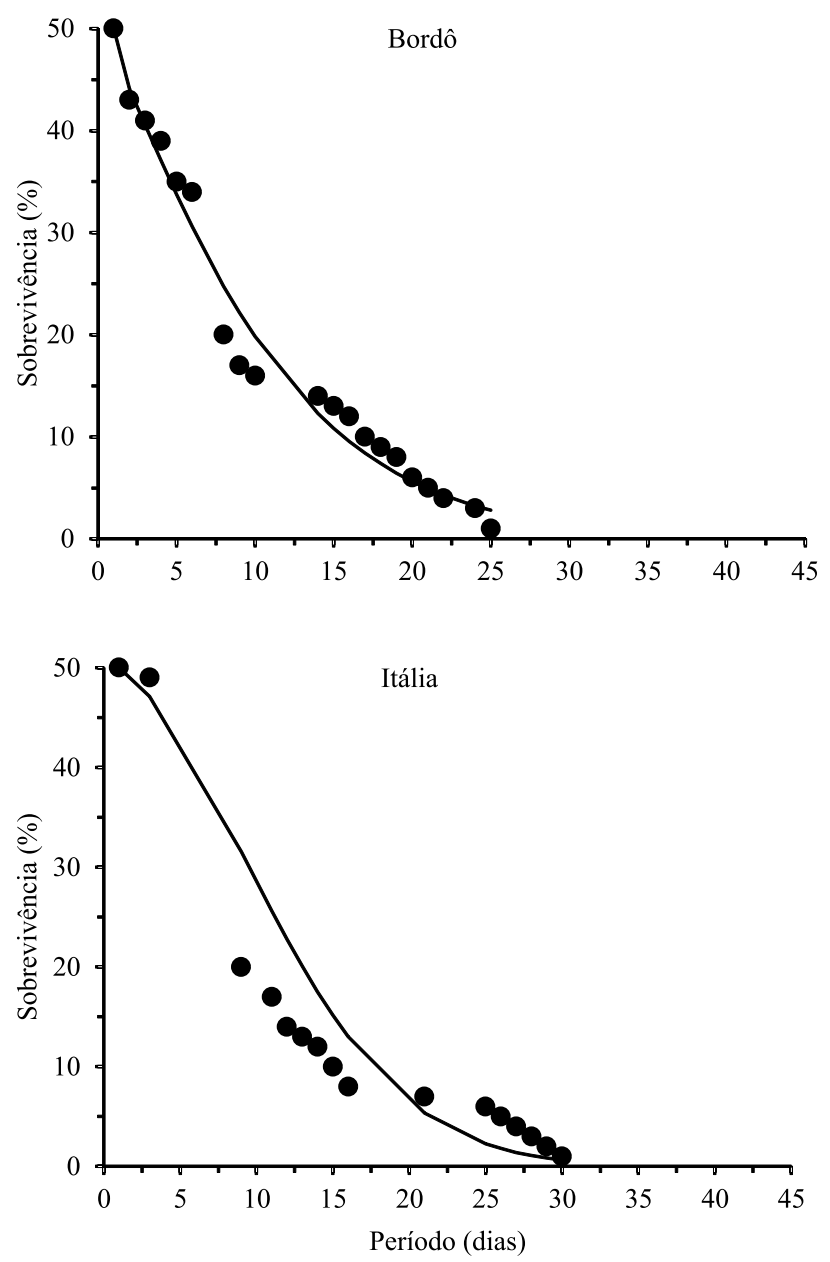

A 'Bordô' afetou negativamente o desenvolvimento biológico do pulgão-preto da videira, tendo proporcionado a menor viabilidade na fase ninfal, menor fecundidade e maior duração do período ninfal, o que resultou em menor potencial de infestação de pulgão-preto. Esta constatação é importante para $o$ manejo deste inseto na cultura da videira, pois, em vinhedos cultivados com a 'Bordô', o potencial de crescimento do pulgão é menor. No entanto, 'Cabernet Franc', 'Itália' e 'Moscato Bianco' favoreceram o desenvolvimento do pulgão-preto e permitiram maior incremento populacional da espécie.

A taxa líquida de reprodução, que representa o número total de ninfas produzidas por fêmea, no decorrer de
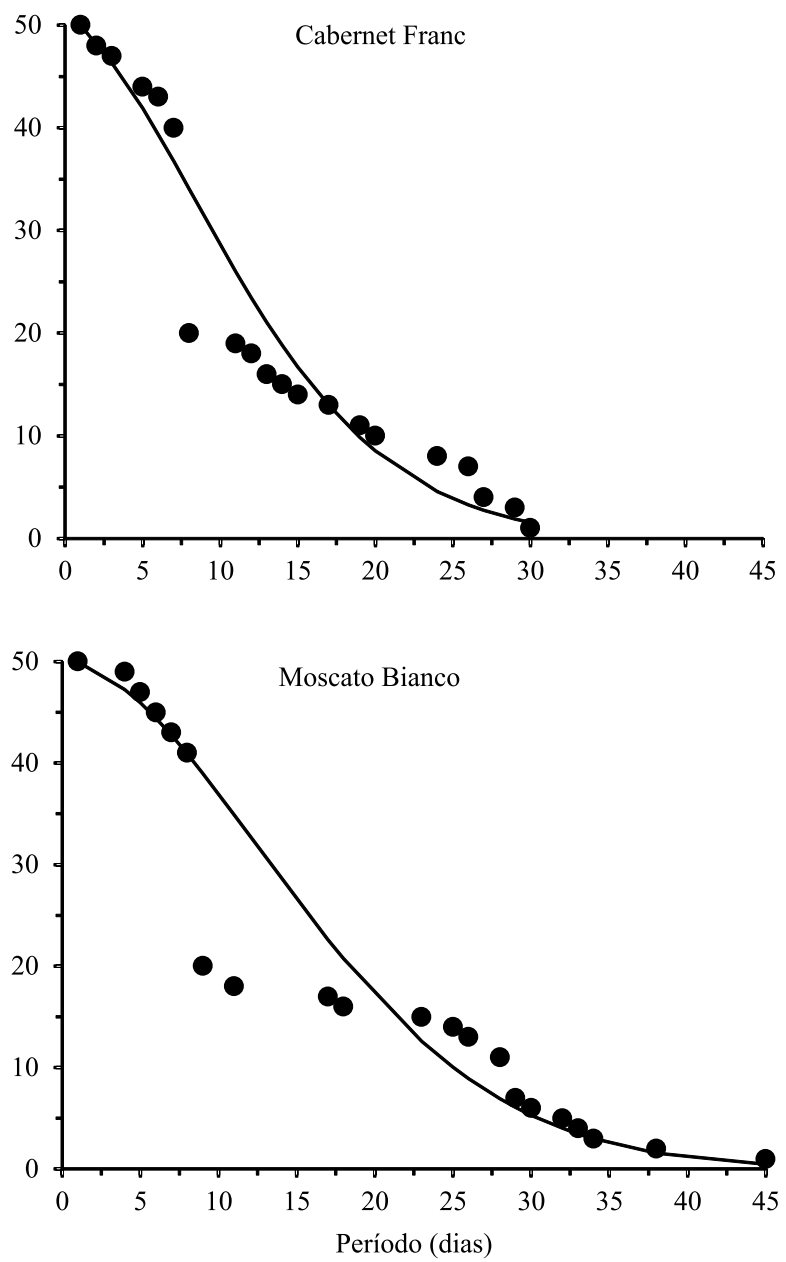

- Sobrevivência observada Sobrevivência esperada

Figura 1. Sobrevivência observada e esperada (Weibull) de fêmeas do pulgão-preto da videira (Aphis illinoisensis), em quatro cultivares de videira, em câmara climatizada do tipo fitotron (a $25 \pm 1^{\circ} \mathrm{C}, 75 \pm 10 \%$ de umidade relativa e fotófase de 14 horas). 
uma geração, apresentou diferença significativa em consequência da cultivar sobre a qual os pulgões se desenvolveram (Tabela 3). Pode-se inferir com estes resultados que o pulgão-preto da videira, a cada geração, cresce 51,30 sobre a 'Cabernet Franc' e 55,60 vezes sobre a 'Moscato Bianco', valores superiores aos das populações que se alimentam da 'Bordô' e da 'Itália', com crescimento de 12,55 e 22,75 vezes, respectivamente. A taxa líquida de reprodução do pulgão-preto, observada em 'Cabernet Franc' e 'Moscato Bianco', é semelhante à observada por Moraiti et al. (2012) para este inseto, com reprodução entre 50 a 61 fêmeas por fêmea por geração, em V. vinifera 'Soultanina', 'Savvatiano', 'Moscato Bianco', 'Agiorgitiko', 'Mavrodafni' e 'Merlot', à temperatura de $23^{\circ} \mathrm{C}$.

O intervalo médio entre gerações foi maior na 'Moscato Bianco' do que na 'Bordô', 'Cabernet Franc' e 'Itália'. $\mathrm{O}$ maior valor de $\mathrm{T}$ é uma indicação de que o contínuo desenvolvimento de populações de pulgão-preto na 'Moscato Bianco' pode resultar em menor número de gerações da praga, em comparação às colônias que se desenvolvem na 'Cabernet Franc' e na 'Itália'.

A taxa intrínseca de crescimento foi de 0,161 na 'Bordô', enquanto na 'Cabernet Franc', 'Itália' e 'Moscato Bianco', a taxa foi de $0,282,0,210$ e 0,231 , respectivamente. No que se refere à razão finita de aumento, verificouse que o crescimento diário da população ou o número de fêmeas adicionadas diariamente à população por cada fêmea foi de aproximadamente 1,17 na 'Bordô' e 1,33, 1,23 e 1,26 na 'Cabernet Franc', 'Itália' e 'Moscato Bianco', respectivamente.

Tabela 3. Intervalo médio entre gerações $(\mathrm{T})$, taxa líquida de reprodução $\left(R_{o}\right)$, taxa intrínseca de crescimento $\left(r_{m}\right)$, razão finita de crescimento $(\lambda)$ e tempo necessário para a população do pulgão-preto da videira (Aphis illinoisensis) duplicar-se em número (DT), em mudas de cultivares de videira, em câmara climatizada $\left(\mathrm{a} 25 \pm 1^{\circ} \mathrm{C}, 75 \pm 10 \%\right.$ de umidade relativa e fotófase de 14 horas) do tipo fitotron ${ }^{(1)}$.

\begin{tabular}{lccccc}
\hline Cultivar & $\mathrm{T}($ dias$)$ & $\mathrm{R}_{\mathrm{o}}$ & $\mathrm{r}_{\mathrm{m}}$ & $\lambda$ & $\mathrm{DT}($ dias$)$ \\
\hline 'Bordô' & $15,71 \mathrm{~b}$ & $12,55 \mathrm{c}$ & $0,1611 \mathrm{c}$ & $1,1748 \mathrm{c}$ & $4,30 \mathrm{a}$ \\
'Cabernet Franc' & $13,94 \mathrm{c}$ & $51,30 \mathrm{a}$ & $0,2825 \mathrm{a}$ & $1,3265 \mathrm{a}$ & $2,45 \mathrm{~b}$ \\
'Itália' & $14,87 \mathrm{c}$ & $22,75 \mathrm{~b}$ & $0,2101 \mathrm{~b}$ & $1,2338 \mathrm{~b}$ & $2,30 \mathrm{c}$ \\
'Moscato Bianco' & $17,42 \mathrm{a}$ & $55,60 \mathrm{a}$ & $0,2306 \mathrm{a}$ & $1,2594 \mathrm{a}$ & $3,00 \mathrm{~b}$ \\
\hline
\end{tabular}

${ }^{(1)}$ Médias seguidas por letras iguais, nas colunas, não diferem pelo teste $\mathrm{T}$, a $5 \%$ de probabilidade.
Os valores da taxa intrínseca de crescimento do presente estudo são inferiores aos observados por Moraiti et al. (2012). Os autores observaram entre 0,47 e 0,50 fêmeas por fêmea por dia que não diferiram entre as cultivares de $V$. vinifera estudadas. O menor tempo necessário para a população duplicar em número foi verificado na 'Itália' (2,30 dias), em comparação à 'Bordô', à 'Cabernet Franc' e à 'Moscato Bianco' (Tabela 3). Moraiti et al. (2012) encontraram tempos necessários para a população duplicar que foram inferiores aos encontrados no presente trabalho, com variação de 1,39 a 1,50 dias nas cultivares de $V$. vinifera. A morfologia foliar afeta a sobrevivência e o desempenho de pulgões em diferentes culturas (Zarpas et al., 2006; Buchman \& Cuddington, 2009) e entre cultivares de uma determinada espécie (Razmjou et al., 2006; Silva et al., 2006; Zarpas et al., 2006; Bayhan, 2009; Obopile \& Ositile, 2010; Bernardi et al., 2012). A face inferior de folhas de $V$. vinifera 'Savvatiano' e 'Mavrodafni' são mais pilosas, em comparação às folhas glabras de 'Soultanina' e de 'Merlot', que apresentam superfície rugosa e com maior resistência (Moraiti et al., 2012).

Embora, no presente estudo, outras cultivares de videira tenham sido utilizadas, a morfologia foliar com presença de pilosidade em folhas de V. labrusca 'Bordô' também pode ter afetado a duração e viabilidade ninfal, a fecundidade e a longevidade do pulgão-preto, o que é designado como efeito de antibiose ou antixenose (Smith, 2005), em comparação às folhas de $V$. vinifera 'Cabernet Franc' e 'Itália', que apresentam superfície sem pilosidade, o que as torna mais favoráveis ao desenvolvimento do inseto.

Não foi localizado outro estudo sobre o desenvolvimento biológico do pulgão-preto da videira em cultivares de importância econômica para o Brasil. As cultivares de videira afetaram os parâmetros duração em dias e de uma geração, a taxa líquida de reprodução, a taxa intrínseca de aumento populacional e a razão finita de aumento do pulgão-preto da videira. Na $V$. labrusca 'Bordô', verificou-se menor potencial de crescimento populacional do pulgão-preto do que nas cultivares de V. vinifera.

No entanto, verificou-se que o inseto se multiplicou em todas as cultivares de videira estudadas. Em razão da recente identificação do pulgão-preto em videiras no Sul do Brasil e dos relatos frequentes de infestações elevadas, pode-se considerar que a espécie apresenta potencial para se transformar em praga-chave, principalmente no início do desenvolvimento da cultura. 


\section{Conclusões}

1. O pulgão-preto da videira (Aphis illinoisensis) completa o ciclo biológico em Vitis labrusca 'Bordô' e $V$. vinifera 'Cabernet Franc', 'Itália' e 'Moscato Bianco'.

2. Vitis vinifera 'Cabernet Franc' é a mais favorável ao crescimento populacional do pulgão-preto da videira e $V$. labrusca 'Bordô' é a menos favorável.

\section{Agradecimentos}

Ao Conselho Nacional de Desenvolvimento Científico e Tecnológico (CNPq), pela concessão de bolsa.

\section{Referências}

AWMACK, C.S.; LEATHER, S.R. Host plant quality and fecundity in herbivorous insects. Annual Review of Entomology, v.47, p.817-844, 2002. DOI: 10.1146/annurev.ento.47.091201.145300.

BARTLETT, M.S. Properties of sufficiency and statistical tests. Proceedings of the Royal Society of London, v.160, p.268-282, 1937. DOI: 10.1098/rspa.1937.0109.

BAYHAN, E. Impact of certain corn cultivars on some biological parameters of Rhopalosiphum maidis (Fitch) (Homoptera: Aphididae). African Journal of Biotechnology, v.8, p.785-788, 2009.

BERNARDI, D.; GARCIA, M.S.; BOTTON, M.; NAVA, D.E. Biology and fertility life table of the green aphid Chaetosiphon fragaefolli on strawberry cultivars. Journal of Insect Science, v.12, p.1-8, 2012. DOI: 10.1673/031.012.2801.

BERTIN, A.; BORTOLI, L.C.; BOTTON, M.; PARRA, J.R.P. Host plant effects on the development, survival, and reproduction of Dysmicoccus brevipes (Hemiptera: Pseudococcidae) on grapevines. Annals of the Entomological Society of America, v.106, p.604-609, 2013. DOI: 10.1603/AN13030.

BLACKMAN, R.L.; EASTOP, V.F. Aphids on the world's herbaceous plants and shrubs: the Natural History Museum. New York: Wiley, 2006. 1439p.

BUCHMAN, N.; CUDDINGTON, K. Influences of pea morphology and interacting factors on pea aphid (Homoptera: Aphididae) reproduction. Environmental Entomology, v.38, p.962-970, 2009. DOI: 10.1603/022.038.0402.

EL-GANTIRY, A.M.; EL-HENEIDY, A.H.; MOUSA, S.F.; ADLY, D. Aphis illinoisensis Shimer (Hemiptera: Aphididae) a recent invasive aphid species in Egypt. Egyptian Journal of Biological Pest Control, v.22, p.225-226, 2012.

FORMOLO, R.; RUFATO, L.; BOTTON, M.; MACHOTA JÚNIOR, R. Diagnóstico da área cultivada com uva fina de mesa (Vitis vinifera L.) sob cobertura plástica e do manejo de pragas. Revista Brasileira de Fruticultura, v.33, p.103-110, 2011. DOI: 10.1590/S0100-29452011005000047.

HARTLEY, H.O. The use of range in analysis of variance. Biometrika, v.37, p.271-280, 1950. DOI: 10.2307/2332380.
HAVELKA, J.; SHUKSHUK, A.H.; GHALIOW, M.E.; LAAMARI, M.; KAVALLIERATOS, N.G.; TOMANOVIC, Z.; RAKHSHANI, E.; PONS, X.; STARY, P. Review of invasive grapevine aphid, Aphis illinoisensis Shimer, and native parasitoids in the Mediterranean (Hemiptera, Aphididae; Hymenoptera, Braconidae, Aphididae). Archives of Biological Sciences, v.63, p.269-274, 2011. DOI: 10.2298/ABS1101269H.

KUNIYUKI, H.; YUKI, V.A.; COSTA, C.L.; COSTA, A.S. No evidence for transmission of three grapevine viruses by the aphid Aphis illinoisensis. Fitopatologia Brasileira, v.20, p.513-514, 1995.

MAIA, A. de H.N.; LUIZ, A.J.B. Programa SAS para análise de tabelas de vida e fertilidade de artrópodes: o método jackknife. Jaguariúna: Embrapa Meio Ambiente, 2006. 11p. (Embrapa Meio Ambiente. Comunicado técnico, 33).

MELLO, L.M.R. de. Vitivinicultura brasileira: panorama 2012. Bento Gonçalves: Embrapa Uva e Vinho, 2013. 5p. (Embrapa Uva e Vinho. Comunicado técnico, 137).

MORAITI, C.A.; MARGARITOPOULOS, J.T.; ZARPAS, K.D.; TSITSIPIS, J.A. The grapevine aphid, Aphis illinoisensis: thermal requirements for development and its performance on six grapevine cultivars. Bulletin of Insectology, v.65, p.29-35, 2012.

OBOPILE, M.; OSITILE, B. Life table and population parameters of cowpea aphid, Aphis craccivora Koch (Homoptera: Aphididae) on five cowpea Vigna unguiculata (L.) Walp. varieties. Journal of Pesticide Science, v.83, p.9-14, 2010. DOI: 10.1007/s10340-0090262-0.

RAZMJOU, J.; MOHARRAMIPOUR, S.; FATHIPOUR, Y.; MIRHOSEINI, S.Z. Demographic parameters of cotton aphid, Aphis gossypii Glover (Homoptera: Aphididae) on five cotton cultivars. Insect Science, v.13, p.205-210, 2006. DOI: 10.1111/j. 1744-7917.2006.00083.x.

SGRILLO, R.B. A distribuição de Weibull como modelo de sobrevivência de insetos. Ecossistema, v.7, p.9-13, 1982.

SHAPIRO S.S.; WILK, M.B. An analysis of variance test for normality (complete samples). Biometrika, v.52, p.591-611, 1965. DOI: 10.1093/biomet/52.3-4.591.

SILVA, A. de A.; VARANDA, E.M.; BAROSELA, J.R. Resistance and susceptibility of alfalfa (Medicago sativa L.) cultivars to the aphid Therioaphis maculata (Homoptera: Aphididae): insect biology and cultivar evaluation. Insect Science, v.13, p.55-60, 2006. DOI: 10.1111/j.1744-7917.2006.00068.x.

SMITH, C.M. Plant resistance to arthropods: molecular and conventional approaches. Netherlands: Springer, 2005. 423p. DOI: 10.1007/1-4020-3702-3.

VALADÃO, G.S.; VIEIRA, M.R.; PIGARI, S.A.A.; TABET, V.G.; SILVA, A.C. da. Resistência de cultivares de videira ao ácaro-rajado Tetranychus urticae na região de Jales, Estado de São Paulo. Revista Brasileira de Fruticultura, v.34, p.1051-1058, 2012. DOI: 10.1590/S0100-29452012000400011.

VENDRAMIN, J.D.; FANCELLI, F. Efeito de genótipos de milho na biologia de Spodoptera frugiperda (J.E. Smith, 1797) (Lepidoptera: Noctuidae). Anais da Sociedade Entomológica do Brasil, v.17, p.141-150, 1988. 
ZA'AQIQ, I.H.M. Field studies on biology, ecology and management of grapevine aphid, Aphis illinoisensis (Shimer) (Homoptera: Aphididae) on some grapevine cultivars Vitis vinifera $\mathbf{L}$. in Al-Arroub Agricultural Experimental Station, Palestine. 2007. 99p. Dissertation (Master) - Hebron University, Hebron.
ZARPAS, K.D.; MARGARITOPOULOS, J.T.; STATHI, L.; TSITSIPIS, J.A. Performance of cotton aphid Aphis gossypii (Hemiptera: Aphididae) lineages on cotton varieties. International Journal of Pest Management, v.52, p.225-232, 2006. DOI: 10.1080/09670870600727305.

Recebido em 15 de abril de 2014 e aprovado em 14 de agosto de 2014 